\title{
Article \\ Annihilation Mechanism of Low-Angle Grain Boundary in Nanocrystalline Metals
}

\author{
Guofeng Zhou ${ }^{1}$, Qishan Huang ${ }^{2}\left(\mathbb{D}\right.$, Yingbin Chen $^{3}{ }^{\circledR}$, Xiongqing $\mathrm{Yu}^{1}$ and Haofei Zhou ${ }^{2, *(\mathbb{D})}$ \\ 1 College of Aerospace Engineering, Nanjing University of Aeronautics and Astronautics, \\ Nanjing 210016, China; zgf02000@aliyun.com (G.Z.); yxq@nuaa.edu.cn (X.Y.) \\ 2 Center for X-Mechanics and State Key Laboratory of Fluid Power and Mechatronic Systems, \\ Department of Engineering Mechanics, Zhejiang University, Hangzhou 310027, China; 11924011@zju.edu.cn \\ 3 Center of Electron Microscopy and State Key Laboratory of Silicon Materials, \\ School of Materials Science and Engineering, Zhejiang University, Hangzhou 310027, China; \\ yingbin_chen@zju.edu.cn \\ * Correspondence: haofei_zhou@zju.edu.cn
}

Citation: Zhou, G.; Huang, Q.; Chen, Y.; Yu, X.; Zhou, H. Annihilation Mechanism of Low-Angle Grain Boundary in Nanocrystalline Metals. Metals 2022, 12, 451. https://doi.org/ $10.3390 /$ met12030451

Academic Editor: Jordi Sort Viñas

Received: 11 January 2022

Accepted: 3 March 2022

Published: 5 March 2022

Publisher's Note: MDPI stays neutral with regard to jurisdictional claims in published maps and institutional affiliations.

Copyright: (c) 2022 by the authors. Licensee MDPI, Basel, Switzerland. This article is an open access article distributed under the terms and conditions of the Creative Commons Attribution (CC BY) license (https:// creativecommons.org/licenses/by/ $4.0 /)$.

\begin{abstract}
Due to the high density of grain boundaries (GBs), nanocrystalline metals possess superior properties, including enhanced strength, work hardening, and fatigue resistance, in comparison to their conventional counterparts. The expectation of GB migration is critical for grain coarsening and GB annihilation in these materials, significantly affecting the polycrystalline network and mechanical behavior. Here, we perform molecular dynamics (MD) simulations on gold (Au) nanocrystals containing multiple parallelly arranged GBs, with a focus on the investigation of annihilation mechanisms of low-angle grain boundaries (LAGBs). It is observed that the shear-coupled motion of LAGBs, consisting of dislocations, gives rise to their preliminary migration with the reduced separation distance between GBs. With subsequent GB motion, the LAGBs encountered with neighboring GBs, and can be annihilated by various mechanisms, including dislocations interpenetration, dislocations interaction, or dislocations absorption, depending on the specific configuration of the neighboring GB. These findings enhance our understanding of GB interactions and shed light on the controlled fabrication of high-performance nanocrystalline metals.
\end{abstract}

Keywords: nanocrystalline metals; grain boundary; plastic deformation; GB annihilation; molecular dynamics simulation

\section{Introduction}

Nanocrystalline materials, with a high percentage of GBs, are widely attractive for various engineering applications due to their enhanced strength and ductility with respect to their coarse-grained polycrystalline counterparts [1,2]. For metallic components subjected to complex mechanical and thermal loading conditions, such as high-speed lightweight aircraft wings in turbulent air [3,4], microstructural stability has to be considered. Although strategies for improving fatigue and corrosion resistance have been proposed [5,6], these materials typically exhibit softening-induced failure, associated with dislocation exhaustion or low-angle grain boundaries (LAGBs) disappearance [1,7,8]. In general, the softening behavior and ductility are strongly related to the deformation mechanisms. Specifically, GB-mediated deformation mechanisms, such as GB sliding [9,10], GB migration [11], or grain rotation [12], are prevalent and at least partly linked with GB annihilation in metallic nanocrystalline materials [13]. A variety of studies have been focused on the mechanical response of individual GBs [14,15], however, the nanocrystalline networks, consisting of multiple GBs, may exhibit different kinetic behaviors [16,17], which was rarely studied. The plastic deformation behavior in polycrystals, involving cooperative motion and interactions of parallel GBs or GB junctions [18,19], plays an important role and must be taken into account in practice. 
GB annihilation, associated with strain softening, was generally observed during deformation and may result from GB motion (particularly migration and/or sliding), dislocation-GB interaction [20], grain rotation [21], self-mechanical annealing or thermallyactivated GB diffusion [22], etc. [23]. If the boundaries move toward each other sufficiently, they can combine to form a different GB, finally giving rise to GB annihilation. Various investigations showed that during the thermal process or cyclic mechanical loading, the GBs began to migrate and annihilate, resulting in a significantly altered GB network [21]. Moreover, grain microstructure vanishes because of the disappearance of a large number of GBs [24]. However, the dynamical atomic process of GB annihilation was still unclear. Through computer simulations, detailed atomistic information about statics and dynamics can be obtained so as to systematically explore the mechanism of GB annihilation.

In this work, we employed MD simulation on Au nanocrystals with two parallelly arranged GBs to investigate the atomistic process of LAGB annihilation. We found that the deformation mechanism that governs the annihilation behavior of the LAGB depends on the initial GB structure. The difference in shear-coupled migration rates between neighboring GBs gives rise to a reduced separation distance between GBs, leading to accelerated GB migration. As the migration continued, the two GBs merged through dislocation interpenetration, interaction, or absorption for different GBs. The annihilation of LAGBs alters GB structures and contributes to grain coalescence. Our findings provide insights into GB-governed plasticity in nanocrystalline materials.

\section{Materials and Methods}

MD simulations were carried out on Au tricrystals with a total of $\sim 140,000$ atoms using Large-scale Atomic/Molecular Massively Parallel Simulator (LAMMPS, Sandia National Laboratories, Albuquerque, NM, USA) [25] and the embedded atom method (EAM) potentials for Au [26]. Cylindrical tricrystal models with a diameter of $10 \mathrm{~nm}$ and a total height of $30 \mathrm{~nm}$ (10 nm height for each grain) were created by constructing three separate crystals with respective crystallographic orientation and joining them along the axial direction. GBs with different misorientations were generated by tilting grains around the $<110>$ axis. Three boundary layers of atoms at the top and bottom of the system were fixed as rigid slabs. The remaining dynamic atoms were allowed to adjust their positions in a Nose-Hoover thermostat at $300 \mathrm{~K}$. Free boundary conditions were applied parallel to the GB plane. To obtain the equilibrated GB structure, the system was relaxed for 20 ps first and energy minimized with a conjugate-gradient algorithm. During the shear loading, samples were under the canonical ensemble (NVT) at $300 \mathrm{~K}$. A constant shear velocity of $\mathrm{v}=1 \mathrm{~m} \mathrm{~s}^{-1}$ parallel to the boundary plane was applied on the rigid slab of the top grain. A velocity profile with a linear gradient from 0 to $1 \mathrm{~m} \mathrm{~s}^{-1}$ was assigned to the dynamic atoms along the axial direction. (Details shown in Supplementary Figure S1) The time step of the MD simulations was $2 \mathrm{fs}$, and the total simulation time was $3 \mathrm{~ns}$. The average vertical displacement of the GB atoms was recorded as the GB migration displacement. Ovito (OVITO GmbH, Germany) [27] was used to visualize the bicrystal model, and the common neighbor analysis was employed to identify the dissociation of the GBs during the simulations. Atoms with FCC, hexagonal close-packed (HCP) and disordered structures were marked in blue, red, and cyan, respectively.

The atomic von-Mises stress is defined as:

$$
\sigma_{\mathrm{von}-\text { Mises }}=\sqrt{1 / 2\left[\left(\sigma_{\mathrm{x}}-\sigma_{\mathrm{y}}\right)^{2}+\left(\sigma_{\mathrm{x}}-\sigma_{\mathrm{z}}\right)^{2}+\left(\sigma_{\mathrm{z}}-\sigma_{\mathrm{y}}\right)^{2}+6\left(\tau_{\mathrm{xy}}^{2}+\tau_{\mathrm{yz}}^{2}+\tau_{\mathrm{xz}}^{2}\right)\right]}
$$

where $\sigma_{\mathrm{x}}, \sigma_{\mathrm{y}}, \sigma_{\mathrm{z}}, \tau_{\mathrm{xy}}, \tau_{\mathrm{yz}}, \tau_{\mathrm{xz}}$ are the six independent components of the Virial stress tensor. The stress tensor components per atom were obtained by dividing the energy by atomic volume during loading. 


\section{Results}

\subsection{Annihilation at Low-Angle Grain Boundaries}

A series of well-defined tri-crystals, comprising two GBs (Figure 1a,b), permits a much more unambiguous study of the structure evolution and further the dynamic deformation mechanisms of GB annihilation in polycrystals. Figure 1c shows a typical relaxed structure with a diameter of $10 \mathrm{~nm}$ and a height of $30 \mathrm{~nm}$. Two [110] tilt GBs (denoted as $\mathrm{GB}_{1}$ and $\mathrm{GB}_{2}$ ) with misorientations of $10^{\circ}$ and $10^{\circ}$ parallel to each other. The signs of misorientations are defined in Figure 1b, where plus represents an anti-clockwise misorientation of the upper grain relative to the corresponding bottom grain and minus is the opposite. Shearing (Figure 1d-f) was imposed on the sample with a direction parallel to the GBs. The structure of the considered low-angle tilt GBs can be represented by a set of periodically located Shockley partial pairs (Figure 1k), thus the migration of GBs is the movement of the wall of the dislocation pairs. Shear loading initially activated GB migration at different rates for $\mathrm{GB}_{1}$ and $\mathrm{GB}_{2}$ because $\mathrm{GB}_{1}$ possessed stronger mobility compared with $\mathrm{GB}_{2}$ (Figure $1 \mathrm{~d}$ ). As this process developed, two GBs approached each other, resulting in a reduction of the separation distance between GBs and shrinkage of G2. While the two GBs got closer and closer, quick merging and annihilation of initial GBs were observed. During the merging process, $\mathrm{GB}_{1}$ instantly moved upward, while $\mathrm{GB}_{2}$ reversed the direction of motion, i.e., move downward, to the same position, leading to a full annihilation of G2 (Figure 1d,e). The newly recombined $\mathrm{GB}_{3}$, as a single wall of dislocations with the misorientation of $20^{\circ}$ (Figure 1k), steadily migrated in the subsequent shear process (Figure 1f).
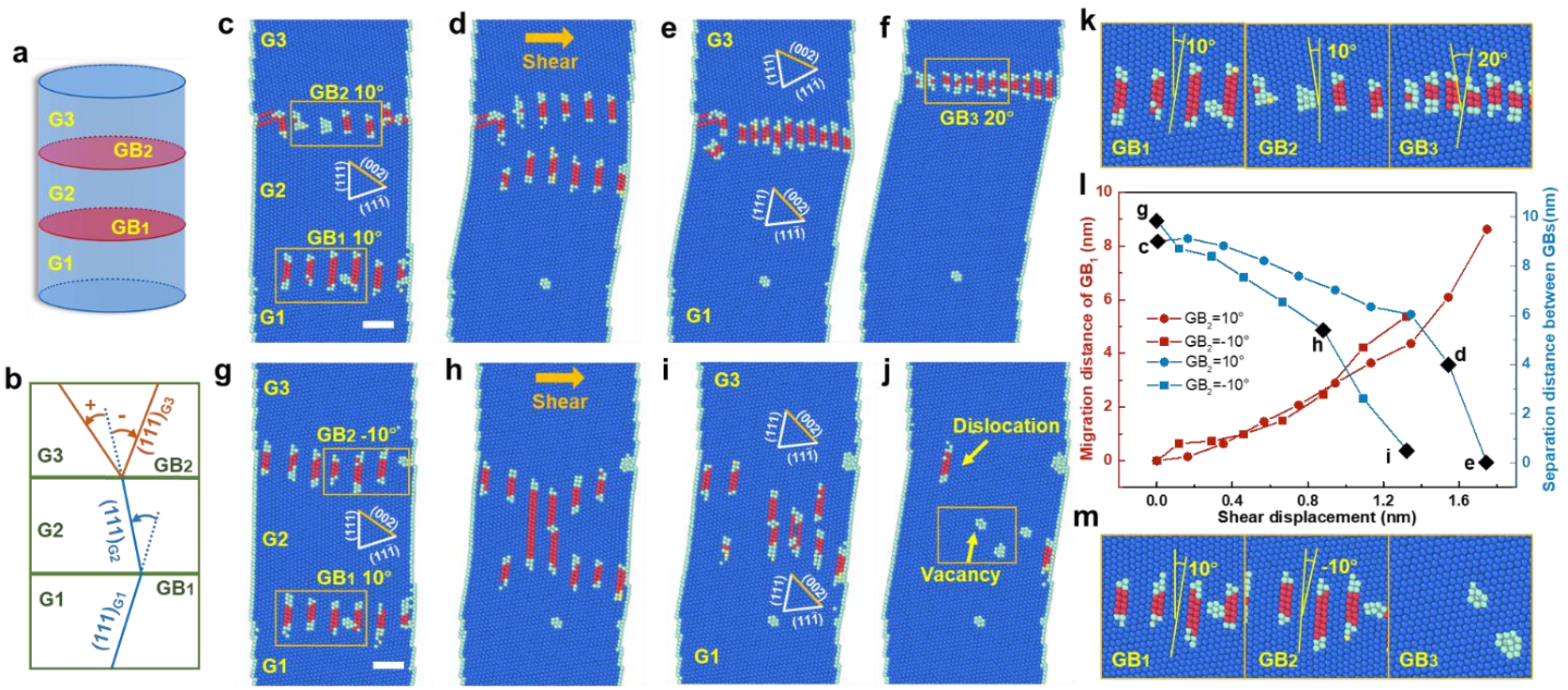

Figure 1. Low-angle grain boundaries (LAGBs) annihilated at LAGBs. (a) Schematic showing the setup of the cylindrical tricrystal sample in this work. (b) The definition of misorientation sign. (c,g) The initial constructed Au nanocrystal, which contained two parallel LAGBs, denoted as $G_{1}$ and $G_{2}$. The misorientations of $\mathrm{GB}_{1}$ is $10^{\circ}$ and of $\mathrm{GB}_{2}$ is $10^{\circ}$ in (c) while $-10^{\circ}$ in (g), respectively. (d) Upon shearing, the two GBs migrated upward at different rates, resulting in a GB spacing reduction. (e) GB dislocation pairs interpenetrated, giving rise to a new GB. (f) The newly formed GB, denoted as $\mathrm{GB}_{3}$, migrated upward in subsequent shearing. (h) Upon shearing, $\mathrm{GB}_{1}$ migrated upward while $\mathrm{GB}_{2}$ migrated downward. (i) While the two GBs meet, GB dislocation interacted. (j) GB annihilation with left vacancies and dislocations. $(\mathbf{k}, \mathbf{m})$ Enlarged structure in $(\mathbf{c}, \mathbf{f}, \mathbf{g}, \mathbf{j})$ showing detailed GB structures before and after annihilation. (1) Migration distance of $\mathrm{GB}_{1}$ and separation distance between two GBs versus shear displacement. The deformation snapshots of $(\mathbf{c}-\mathbf{e}, \mathbf{g}-\mathbf{i})$ were highlighted by black diamonds. Atoms with FCC, HCP and disordered structures were marked in blue, red, and cyan, respectively. Scale bars: $2 \mathrm{~nm}$. 
To further explore the annihilation mechanisms between LAGBs, additional MD simulation was carried out by altering the misorientation of $\mathrm{GB}_{2}$ (Figure $1 \mathrm{~b}$ ), while fixing the misorientation of $\mathrm{GB}_{1}$. For $\mathrm{GB}_{2}$ with a misorientation of $-10^{\circ}$, the $\mathrm{G} 1$ and $\mathrm{G} 3$ possess the same orientation but with some shifting/transition along the GB plane (Figure $1 \mathrm{~g}, \mathrm{~m}$ ). Upon shearing, the two GBs moved toward each other, engendering the shrinkage of G2 (Figure 1h). As the GB closer to another one, the interaction force between neighboring disassociated dislocation partials grew rapidly. Interaction of dislocations in the same slip planes generated an elongated extended dislocation (Figure 1h). Subsequently, the extended dislocations shrank instantly and finally annihilated (Figure 1i). We found the initial straight GBs became convexly curved towards G2 during migration, probably induced by the non-uniform distribution of stress at the GBs and the annihilation of the internal GB dislocations relaxed stresses, while the stationery boundary GB dislocations fixed the GB. After dislocation pairs interaction and fully annihilation, a single crystal formed with some left-behind vacancies and residual dislocations (Figure 1j,m).

During the above shearing process, GB positions were recorded, and both migration distance of $\mathrm{GB}_{1}$ and separation distance between $\mathrm{GB}_{1}$ and $\mathrm{GB}_{2}$ were measured (Figure 11, details shown in Supplementary Tables S1 and S2). Attention was first focused on the initial migration of $\mathrm{GB}_{1}$ for models with different $\mathrm{GB}_{2}$, identical and unchanged migration-shear displacement coupling were shown during their motion till the time moments close to GB meeting. The coupling factors were estimated to be 0.24 , somewhat larger than the theoretical value calculated based on the shear migration geometrical model [15]:

$$
\beta=2 \tan \left(\frac{\theta}{2}\right) \sim 0.175
$$

The variation between simulation and theory may result from temperature [14], free surface [28], GB structure [15], etc. Then, a sharp transition of GB migration rates was found at a separation distance of $\sim 6 \mathrm{~nm}$, which suggests the mutual attraction between GBs effectively accelerated the merging process. In the case of FCC materials, the annihilation distance between edge dislocations can be approximated as $6 \boldsymbol{b}$, where $\boldsymbol{b}$ is the Burgers vector of dislocations [29] ( $\sim 3 \mathrm{~nm}$ for disassociated dislocations). The difference may be induced by lateral dislocations, stress, or temperature.

Dislocation analysis and stress contribution evolution observations were further carried out to explore detailed atomic dynamics during annihilation. For $\mathrm{GB}_{2}$ with the misorientation of $10^{\circ}$, the Burgers vectors of GB dislocations were $\boldsymbol{b}_{1}=1 / 6[-1-2-1]$, $\boldsymbol{b}_{2}=1 / 6[1-1-2]$, while the Burgers vectors of dislocations, compromised of $\mathrm{GB}_{1}$, were $\boldsymbol{b}_{3}=1 / 6[-1-2-1], \boldsymbol{b}_{4}=1 / 6[1-1-2]$ (Figure 2a). Stress contribution showed severe concentration at $\mathrm{GB}_{2}$ while highly mobile $\mathrm{GB}_{1}$ migrated to partly release stress (Figure 2e). The increasing attraction with the reduction of GBs separation distance accelerated the migration of $\mathrm{GB}_{1}$ and reversed the migration direction of $\mathrm{GB}_{2}$ (Figure $2 \mathrm{~b}$ ). Dislocations spacing of $\mathrm{GB}_{1}$ and $\mathrm{GB}_{2}$ were initially four (111) atom layers. After dislocations crossing (Figure 2b), dislocation spacing turned to be one (111) atom layer (Figure 2c). Accompanied with the shrinkage of stacking faults (Figure 2d), stress was released in G2 and concentrated at $\mathrm{GB}_{3}$ to further activate the migration of $\mathrm{GB}_{3}$ (Figure $2 \mathrm{f}$ ).

For $\mathrm{GB}_{2}$ with the misorientation of $-10^{\circ}$, the Burgers vectors of $\mathrm{GB}$ dislocations were,

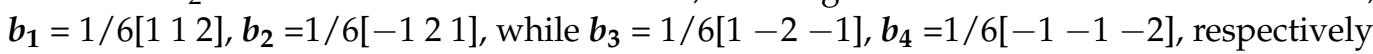
(Figure $2 \mathrm{~g}$ ). A perfect annihilation occurred to the opposite dislocation pairs $\left(\boldsymbol{b}_{2}=-\boldsymbol{b}_{3}\right)$, locating at the same slip planes. In contrast, the annihilation of the dislocation pairs at the adjacent planes generated some vacancies at the meeting area (Figure $2 \mathrm{~h}$ ). Immediate annihilation of trailing partials occurred with stacking fault eliminated, inducing the merging of $\mathrm{GB}_{1}$ and $\mathrm{GB}_{2}$ and further coalescence of G1 and G3 (Figure 2i). However, dislocations near the surface cannot be removed and would glide in subsequent deformation (Figure 2j). Due to the annihilation of GBs, stress was released and re-concentrated at the residual vacancies and dislocations (Figure 2k,l). 

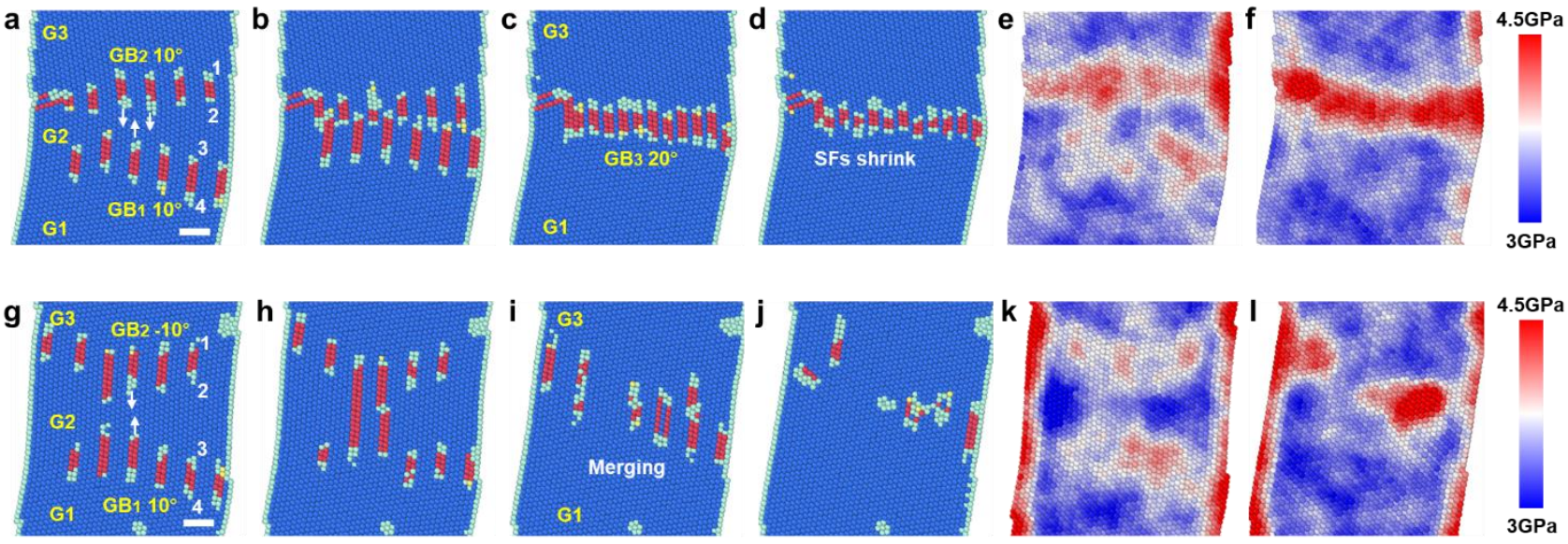

Figure 2. Atomistic dynamics and stress contribution evolution during LAGBs annihilated at LAGBs. (a-d) Simulation snapshots showing $\mathrm{GB}_{1}$, with a misorientation of $10^{\circ}$, annihilated at $\mathrm{GB}_{2}$, with a misorientation of $10^{\circ}$. (a) The two LAGBs consist of disassociated dislocation pairs, denoted as dislocations 1-4. (b,c) Dislocation pairs glided and interpenetrated into the gaps. (d) After the dislocation pairs are arranged in a row, the stacking faults shrink. (e,f) The atomic von-Mises stress contribution of $(\mathbf{a}, \mathbf{d})$, showing increased stress concentration at GBs after GB annihilation. $(\mathbf{g}-\mathbf{j})$ Simulation snapshots showing $\mathrm{GB}_{1}$, with a misorientation of $10^{\circ}$, annihilated at $\mathrm{GB}_{2}$, with a misorientation of $-10^{\circ}$. (g) The two LAGBs consist of disassociated dislocation pairs, denoted as dislocations 1-4. (h,i) Opposite dislocation partials interacted, and the two GBs merged. (j) While $\mathrm{GB}_{1}$ fully annihilated, there left some dislocations and vacancies. $(\mathbf{k}, \mathbf{l})$ The atomic von-Mises stress contribution of $(\mathrm{g}, \mathbf{j})$, showing increased stress concentration at vacancies after GB annihilation. Scale bars: $2 \mathrm{~nm}$.

\subsection{Annihilation at High-Angle Grain Boundaries}

High-angle grain boundaries (HAGBs), with low mobility, act as a barrier for the dislocation glide, leading to a dislocation impediment [30]. To understand the mechanism of the impediment and annihilation of LAGBs at HAGBs, we carried out additional simulations for $\mathrm{GB}_{2}$ with a misorientation of $30^{\circ}$ (Figure $3 \mathrm{a}$ ) and $-30^{\circ}$ (Figure 3e). During the whole shearing process, $\mathrm{GB}_{2}$ stayed at the initial positions while $\mathrm{GB}_{1}$ migrated upward and approached $\mathrm{GB}_{2}$ (Figure $3 \mathrm{~b}, \mathrm{f}$ ). With continued loading, the leading partials (Figure $3 \mathrm{i}, \mathrm{k}$ ) were absorbed and trailing partials were attached to $\mathrm{GB}_{2}$ with stacking-fault ribbons connecting (Figure $3 \mathrm{c}, \mathrm{g}$ ). Eventually, $\mathrm{GB}_{2}$ absorbed all dislocation pairs, i.e., $\mathrm{GB}_{1}$. The rearrangement of atoms at $\mathrm{GB}_{2}$ resulted in $\mathrm{GB}$ structures modification and generating new GBs (Figure 3i,k). The newly formed $\mathrm{GB}_{3}$ were still immobile during the subsequent shearing (Figure $3 \mathrm{~d}, \mathrm{~h}$ ). Quantitative measurement showed a consistent initial coupling factor of 0.22 and critical transition GB separation distance of $\sim 5 \mathrm{~nm}$ (Figure 3j, details shown in Supplementary Tables S3 and S4).

Detailed GB annihilation processes and stress contributions are shown in Figure 4. The un-uniform migration of $\mathrm{GB}_{1}$ resulted in curved GB structures (Figure 4a,g) and different local separation distances between $\mathrm{GB}_{2}$ and $\mathrm{GB}_{1}$ segments. The immobile $\mathrm{GB}_{2}$ bore much more stress concentration compared with $\mathrm{GB}_{1}$ (Figure 4e,k). In response to the localized stress at $\mathrm{GB}_{2}$, the foremost leading partial dislocation annihilated and dissociated into GB dislocations at $\mathrm{GB}_{2}$, leaving the corresponding trailing partial attached to $\mathrm{GB}_{2}$ (Figure $4 \mathrm{~b}, \mathrm{~h}$ ). Then, the trailing partial glided into $\mathrm{GB}_{2}$ and annihilated at $\mathrm{GB}_{2}$, accompanied with other leading partials approached $\mathrm{GB}_{2}$ (Figure $4 \mathrm{c}, \mathrm{i}$ ). With all dislocation pairs, compromised of $\mathrm{GB}_{1}$, annihilated at $\mathrm{GB}_{2}$ one by one, the structures of HAGBs were altered with increasing or decreasing misorientations (Figure $4 \mathrm{~d}, \mathrm{j}$ ). Stress concentrated severely at $\mathrm{GB}_{3}$ and free surface, while completely released in G1 (Figure 4f,l). 

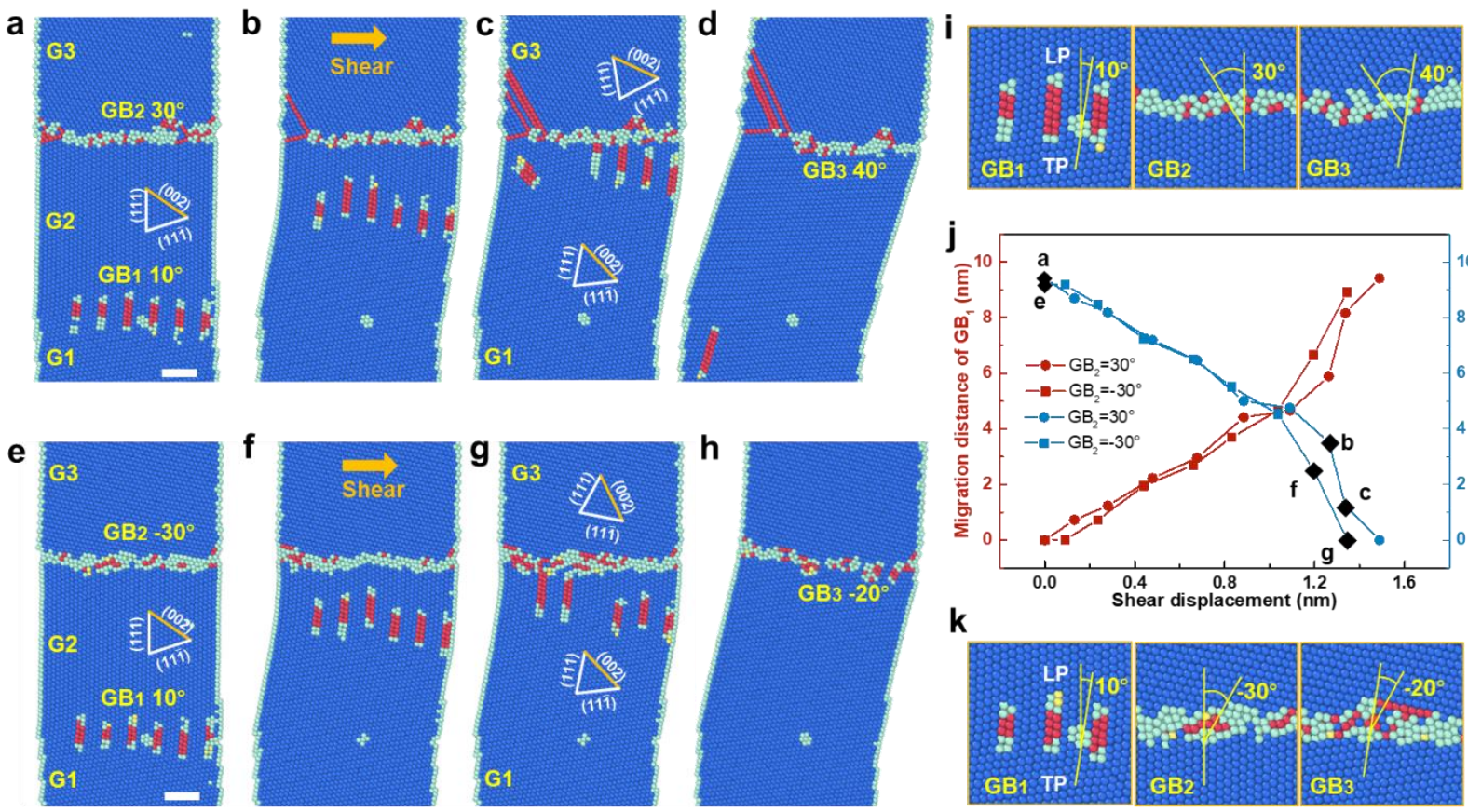

Figure 3. LAGBs annihilated at high-angle grain boundaries (HAGBs). (a,e) The initial constructed Au nanocrystal contained two parallel GBs. The misorientation of the low-angle GB, denoted as $\mathrm{GB}_{1}$, was $10^{\circ}$ and the high-angle $\mathrm{GB}$, denoted $\mathrm{as}_{2} \mathrm{~GB}_{2}$, was $30^{\circ}$ in $(\mathbf{a})$, while $-30^{\circ}$ in (e), respectively. $(\mathbf{b}, \mathbf{f})$ Upon shearing, $\mathrm{GB}_{1}$ migrated upward and approached $\mathrm{GB}_{2}$, resulting in a $\mathrm{GB}$ spacing reduction. (c, $\mathbf{g}$ ) The leading partials, denoted as LP in (i,k), were absorbed, leaving the trailing partials, denoted as TP in $(\mathbf{i}, \mathbf{k})$, attached to $\mathrm{GB}_{2}$ with stacking-fault ribbons. (d,h) $\mathrm{GB}_{1}$ fully annihilated at $\mathrm{GB}_{2}$, generating new GBs, denoted as $\mathrm{GB}_{3}$. (i,k) Enlarged structure in $(\mathbf{a}, \mathbf{d}, \mathbf{e}, \mathbf{h})$ showing detailed GB structures before and after annihilation. (j) Migration distance of $\mathrm{GB}_{1}$ and separation distance between two GBs versus shear displacement. The deformation snapshots of $(\mathbf{a}-\mathbf{c}, \mathbf{e}-\mathbf{g})$ were highlighted by black diamonds. Scale bars: $2 \mathrm{~nm}$.
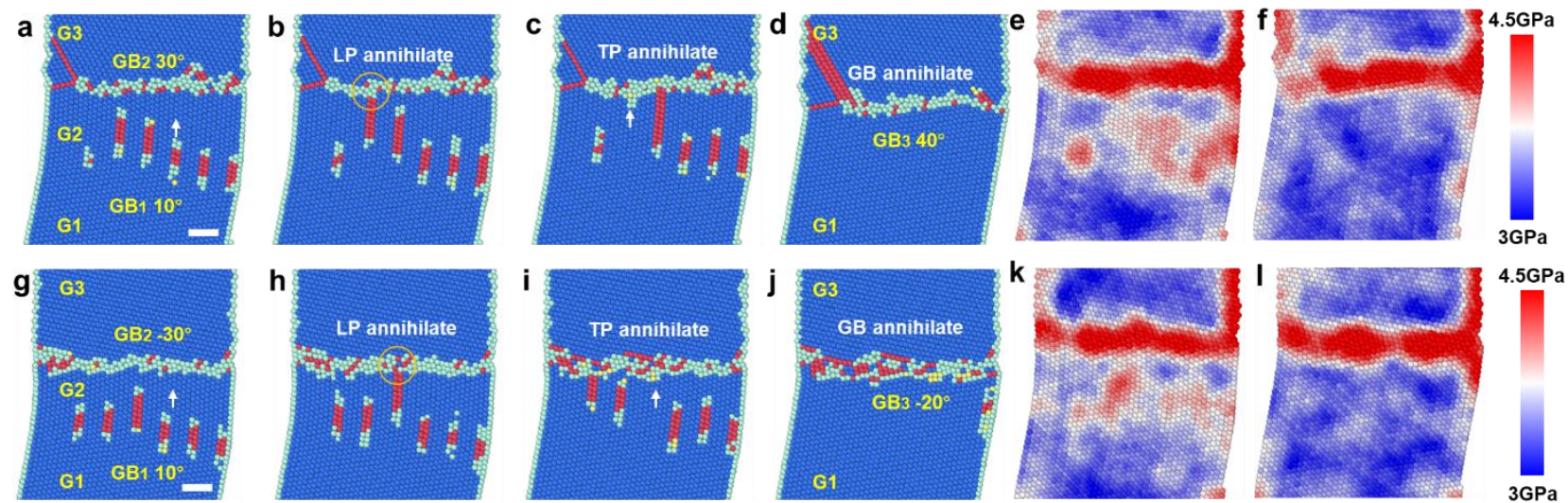

Figure 4. Atomistic dynamics and stress contribution evolution during LAGBs annihilated at HAGBs. $(\mathbf{a}-\mathbf{d}, \mathbf{g}-\mathbf{j})$ Simulation snapshots showing $\mathrm{GB}_{1}$, with a misorientation of $10^{\circ}$, annihilated at $\mathrm{GB}_{2}$, with a misorientation of $30^{\circ}$ and $-30^{\circ}$, respectively. (a,g) The LAGB $\left(G_{1}\right)$ consists of disassociated dislocation pairs, which brings about high mobility. (b,h) Leading partials of $\mathrm{GB}_{1}$ annihilated at $\mathrm{GB}_{2}$, leaving the corresponding trailing partials attached to $\mathrm{GB}_{2}$. (c,i) The trailing partials glided into $\mathrm{GB}_{2}$ and annihilated. $(\mathbf{d}, \mathbf{j})$ While all dislocation pairs at $\mathrm{GB}_{2}$, the LAGB $\left(\mathrm{GB}_{1}\right)$ fully annihilated at $\mathrm{GB}_{2}$. $(\mathbf{e}, \mathbf{k})$ The atomic von-Mises stress contribution of (a) and (g), showing severe stress concentration at $\mathrm{GB}_{2}$ and slight stress concentration at $\mathrm{GB}_{1}$ before $\mathrm{GB}$ annihilation. (f,l) The atomic von-Mises stress contribution of $(\mathbf{d}, \mathbf{j})$, showing released stress in G1 after GB annihilation. Scale bars: $2 \mathrm{~nm}$. 
Taken together, these results provide important insights into the annihilation mechanisms of LAGBs, which are strongly dependent on the GB structures. Further quantitative analysis, including migration distance of $\mathrm{GB}_{1}$ and separation distance between two GBs, suggested an almost consistent migration rate of $\mathrm{GB}_{1}$ and critical accelerating separation spacings between GBs before meeting, which can give deep insight into GB-dominated plasticity.

\section{Discussion}

With applied force, the motion of GBs within a polycrystal is inevitably impeded by other grains and GB junctions, generally generating grain rotation. Multiple grain rotations bring the orientation of abutting grains closer together, which reduces the GB misorientation angles and even eliminates the GBs, leading to the coalescence of smaller grains [12,21,31]. However, previous observations also showed that LAGBs were activated to migrate steadily before being annihilated [32,33], different from grain rotation mediated GB annihilation. Moreover, the shear-coupled GB migration was believed to have contributed to grain shrinkage and GB annihilation [11,34,35]. The shrinkage of grain, accompanied by the simultaneous growth of neighboring grains, is observed to occur commonly in various materials during plastic deformation under tension [36], shear [11], or indentation [37], finally resulting in statistical grain coarsening. Here, our results showed the high mobility of LAGBs may contribute to a GB annihilation and preferential growth/shrinkage of the corresponding grains.

Stress-assisted migration behavior of individual LAGBs was widely studied. LAGBs were traditionally described in terms of dislocation arrays, and shear stresses were generally thought to influence and activate LAGBs by coupling to the individual dislocations in these arrays $[32,38,39]$. However, the deformation dynamics of polycrystals, consisting of multiple embedded GBs, especially the coordinated behavior between GBs, are still unknown. With a tri-crystal model system, we studied the annihilation behavior of LAGBs at neighboring GBs, including LAGBs (Figures 1 and 2) and HAGBs (Figures 3 and 4). Grain and GB annihilation have resulted from inconsistent structure-dependent shear-coupled migration rates of GBs. During the encountering annihilation process, dislocation pairs interpenetrated and arranged in a row or merged at LAGBs with leaving-behind defects, while they were absorbed one by one at HAGBs (Figure 5).

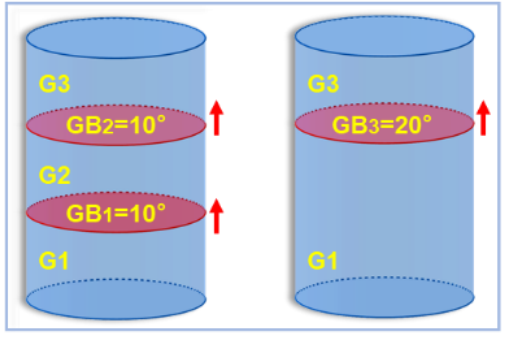

GBs interpenetrating

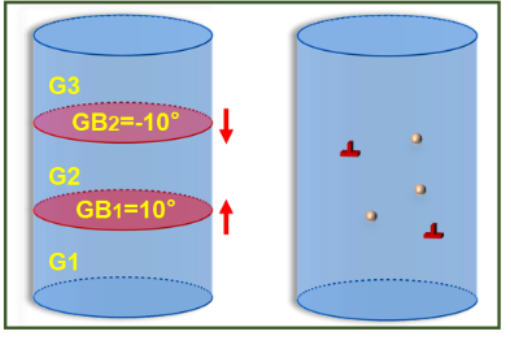

GBs interaction

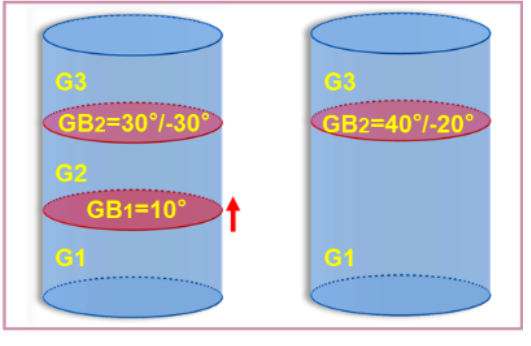

GBs absorption

Figure 5. Schematic showing the annihilation mechanisms of LAGBs depending on the specific configuration of the neighboring GBs. The GBs are found to merge and finally annihilate through dislocation interpenetrating for LAGBs with the same migration direction initially. GBs annihilated through dislocation interaction for LAGBs with the opposite migration direction initially. The left vacancy and residual dislocations are indicated by dots and ' $\mathrm{T}$ '. Meeting with HAGBs, LAGBs annihilated through GBs absorption. Red arrows at the right of GB planes indicate the migration direction under right shear.

Apart from GB structures, other factors should be taken into account to describe proper GB annihilation in polycrystals. For instance, GBs are always dragged by junctions or impurities and cannot migrate until the junctions or impurities are unpinned $[40,41]$. Especially in high defective metals, the pre-existing defects, such as vacancies and intersti- 
tials, will cause jogs formation or the distortion of planes and further alter the deformation mechanisms [42,43]. Furthermore, grain rotation is a deformation mechanism related to GB annihilation, which is often observed to occur during plastic deformation. The coupling effect between grain rotation and GB migration can be particularly important to examine. More investigations should also be carried out in future work on various structures of GBs under different loading conditions.

\section{Conclusions}

In summary, atomic-scale annihilation mechanisms of LAGBs encountering neighboring GBs with applied shearing stress have been studied in Au tri-crystalline models using MD simulations. It is revealed that LAGB kept a consistent migration rate in the early stage. As the separation distance between the GBs decreases, the migration of the LAGB possessing higher mobility accelerates. The GBs are found to merge and finally annihilate through dislocation interpenetrating and interaction for LAGBs or absorption for HAGBs (Figure 5). LAGBs annihilated at neighboring GBs alter the GB network and contribute to grain coalescence. The motion and annihilation of GBs observed in our simulation are calling for experiments, such as TEM studies on GB annihilation. These findings may provide insights into the control of polycrystalline networks in nanocrystalline metals further assist the design of reliable metallic nanocomponents for high-performance nanodevices.

Supplementary Materials: The following supporting information can be downloaded at: https: / / www.mdpi.com/article/10.3390/met12030451/s1, Figure S1: Schematic showing the set-up and loading profile.; Table S1: Migration distance of GB1 and separation distance between two GBs versus shear displacement for $10^{\circ}$ LAGB annihilated at $10^{\circ}$ LAGB; Table S2: Migration distance of GB1 and separation distance between two GBs versus shear displacement for $10^{\circ}$ LAGB annihilated at $-10^{\circ}$ LAGB; Table S3: Migration distance of GB1 and separation distance between two GBs versus shear displacement for $10^{\circ}$ LAGB annihilated at $30^{\circ}$ HAGB; Table S4: Migration distance of GB1 and separation distance between two GBs versus shear displacement for $10^{\circ} \mathrm{LAGB}$ annihilated at $-30^{\circ} \mathrm{HAGB}$;

Author Contributions: Conceptualization, H.Z.; Data curation, Q.H. and X.Y.; Formal analysis, G.Z. and Y.C.; Investigation, G.Z., Q.H. and X.Y.; Methodology, Q.H.; Project administration, H.Z.; Validation, X.Y.; Visualization, Q.H.; Writing-original draft, G.Z., Q.H., Y.C. and H.Z.; Writing-review \& editing, G.Z., Q.H., X.Y. and H.Z. All authors contributed to the data analysis and paper revision. All authors have read and agreed to the published version of the manuscript.

Funding: This research was funded by the National Natural Science Foundation of China, grant number $11902289,12172324$.

Institutional Review Board Statement: Not applicable.

Informed Consent Statement: Not applicable.

Data Availability Statement: The data that support the findings of this study are available from the corresponding author upon reasonable request.

Conflicts of Interest: The authors declare no conflict of interest.

\section{References}

1. Hu, J.; Shi, Y.N.; Sauvage, X.; Sha, G.; Lu, K. Grain boundary stability governs hardening and softening in extremely fine nanograined metals. Science 2017, 355, 1292-1296. [CrossRef] [PubMed]

2. Zhu, T.; Li, J. Ultra-strength materials. Prog. Mater. Sci. 2010, 55, 710-757. [CrossRef]

3. Srinivasan, V.; Kunjiappan, S.; Palanisamy, P. A brief review of carbon nanotube reinforced metal matrix composites for aerospace and defense applications. Int. Nano Lett. 2021, 11, 321-345. [CrossRef]

4. Divagar, S.; Vigneshwar, M.; Selvamani, S.T. Impacts of Nano Particles on Fatigue Strength of Aluminum Based Metal Matrix Composites for Aerospace. Mater. Today Proc. 2016, 3, 3734-3739. [CrossRef]

5. Pan, Q.; Zhou, H.; Lu, Q.; Gao, H.; Lu, L. History-independent cyclic response of nanotwinned metals. Nature 2017, 551, 214-217. [CrossRef] 
6. Ghosh, D.; Shukla, A.K.; Roy, H. Nano Structured Plasma Spray Coating for Wear and High Temperature Corrosion Resistance Applications. J. Inst. Eng. (India) Ser. D 2014, 95, 57-64. [CrossRef]

7. Li, X.; Wei, Y.; Lu, L.; Lu, K.; Gao, H. Dislocation nucleation governed softening and maximum strength in nano-twinned metals. Nature 2010, 464, 877-880. [CrossRef]

8. Schiotz, J.; Tolla, F.; Jacobsen, K.W. Softening of nanocrystalline metals at very small grain sizes. Nature 1998, 391, 561-563. [CrossRef]

9. Langdon, T.G. Grain boundary sliding revisited: Developments in sliding over four decades. J. Mater. Sci. 2006, 41, 597-609. [CrossRef]

10. Li, Q.; Wang, L.; Teng, J.; Pang, X.; Zou, J. In-situ observation of cooperative grain boundary sliding and migration in the nano-twinned nanocrystalline-Au thin-films. Scr. Mater. 2020, 180, 97-102. [CrossRef]

11. Thomas, S.L.; Chen, K.; Han, J.; Purohit, P.K.; Srolovitz, D.J. Reconciling grain growth and shear-coupled grain boundary migration. Nat. Commun. 2017, 8, 1764. [CrossRef] [PubMed]

12. Wang, L.; Teng, J.; Liu, P.; Hirata, A.; Ma, E.; Zhang, Z.; Chen, M.; Han, X. Grain rotation mediated by grain boundary dislocations in nanocrystalline platinum. Nat. Commun. 2014, 5, 4402. [CrossRef] [PubMed]

13. Kumar, K.S.; Van Swygenhoven, H.; Suresh, S. Mechanical behavior of nanocrystalline metals and alloys. Acta Mater. 2003, 51, 5743-5774. [CrossRef]

14. Cahn, J.W.; Mishin, Y.; Suzuki, A. Coupling grain boundary motion to shear deformation. Acta Mater. 2006, 54, $4953-4975$. [CrossRef]

15. Homer, E.R.; Foiles, S.M.; Holm, E.A.; Olmsted, D.L. Phenomenology of shear-coupled grain boundary motion in symmetric tilt and general grain boundaries. Acta Mater. 2013, 61, 1048-1060. [CrossRef]

16. Wang, P.; Yang, X.; Peng, D. Molecular dynamics investigation of the grain boundary migration hysteresis of nanocrystalline Ni under cyclic shear loading. Modell. Simul. Mater. Sci. Eng. 2017, 25, 025006. [CrossRef]

17. Luo, X.M.; Zhang, B.; Zhu, X.F.; Zhou, Y.T.; Xiao, T.Y.; Zhang, G.P. Local-structure-affected behavior during self-driven grain boundary migration. MRS Commun. 2016, 6, 85-91. [CrossRef]

18. Yu, T.; Hughes, D.A.; Hansen, N.; Huang, X. In situ observation of triple junction motion during recovery of heavily deformed aluminum. Acta Mater. 2015, 86, 269-278. [CrossRef]

19. Ovid'ko, I. Triple junction nanocracks in deformed nanocrystalline materials. Acta Mater. 2004, 52, 1201-1209. [CrossRef]

20. Kacher, J.; Eftink, B.P.; Cui, B.; Robertson, I.M. Dislocation interactions with grain boundaries. Curr. Opin. Solid State Mater. Sci. 2014, 18, 227-243. [CrossRef]

21. Dake, J.M.; Oddershede, J.; Sorensen, H.O.; Werz, T.; Shatto, J.C.; Uesugi, K.; Schmidt, S.; Krill, C.E., III. Direct observation of grain rotations during coarsening of a semisolid Al-Cu alloy. Proc. Natl. Acad. Sci. USA 2016, 113, E5998-E6006. [CrossRef] [PubMed]

22. Aleshin, A.N. Role of grain-boundary diffusion in the grain growth in nanocrystalline nickel. Russ. Metall. (Metally) 2008, 2008, 286-293. [CrossRef]

23. Ni, H.; Zhu, J.; Wang, Z.; Lv, H.; Su, Y.; Zhang, X. A brief overview on grain growth of bulk electrodeposited nanocrystalline nickel and nickel-iron alloys. Rev. Adv. Mater. Sci. 2019, 58, 98-106. [CrossRef]

24. Sauzay, M.; Brillet, H.; Monnet, I.; Mottot, M.; Barcelo, F.; Fournier, B.; Pineau, A. Cyclically induced softening due to low-angle boundary annihilation in a martensitic steel. Mater. Sci. Eng. A 2005, 400-401, 241-244. [CrossRef]

25. Plimpton, S. Fast Parallel Algorithms for Short-Range Molecular Dynamics. J. Comp. Phys. 1995, 117, 1-19. [CrossRef]

26. Grochola, G.; Russo, S.P.; Snook, I.K. On fitting a gold embedded atom method potential using the force matching method. J. Chem. Phys. 2005, 123, 204719. [CrossRef]

27. Stukowski, A. Visualization and analysis of atomistic simulation data with OVITO-The Open Visualization Tool. Modell. Simul. Mater. Sci. Eng. 2010, 18, 015012. [CrossRef]

28. Pan, J.; Cocks, A.; Kucherenko, S. Finite element formulation of coupled grain-boundary and surface diffusion with grainboundary migration. Proc. R. Soc. A Math. Phys. Eng. Sci. 1997, 453, 2161-2184. [CrossRef]

29. Wang, H. On the annihilation of dislocation dipoles in metals. AIMS Mater. Sci. 2017, 4, 1231-1239. [CrossRef]

30. Kondo, S.; Mitsuma, T.; Shibata, N.; Ikuhara, Y. Direct observation of individual dislocation interaction processes with grain boundaries. Sci. Adv. 2016, 2, e1501926. [CrossRef]

31. Yuk, J.M.; Jeong, M.; Kim, S.Y.; Seo, H.K.; Kim, J.; Lee, J.Y. In situ atomic imaging of coalescence of Au nanoparticles on graphene: Rotation and grain boundary migration. Chem. Commun. 2013, 49, 11479-11481. [CrossRef] [PubMed]

32. Fomin, E.V.; Mayer, A.E. Slip of low-angle tilt grain boundary (110) in FCC metals at perpendicular shear. Int. J. Plast. 2020, 134, 102843. [CrossRef]

33. Bishop, G.H.; Harrison, R.J.; Kwok, T.; Yip, S. Computer molecular dynamics simulation studies of grain-boundary structures. II. Migration, sliding, and annihilation in a two-dimensional solid. J. Appl. Phys. 1982, 53, 5609-5616. [CrossRef]

34. Mompiou, F.; Legros, M.; Radetic, T.; Dahmen, U.; Gianola, D.S.; Hemker, K.J. In situ TEM observation of grain annihilation in tricrystalline aluminum films. Acta Mater. 2012, 60, 2209-2218. [CrossRef]

35. Jin, M.; Minor, A.M.; Stach, E.A.; Morris, J.W. Direct observation of deformation-induced grain growth during the nanoindentation of ultrafine-grained $\mathrm{Al}$ at room temperature. Acta Mater. 2004, 52, 5381-5387. [CrossRef] 
36. Gianola, D.S.; Van Petegem, S.; Legros, M.; Brandstetter, S.; Van Swygenhoven, H.; Hemker, K.J. Stress-assisted discontinuous grain growth and its effect on the deformation behavior of nanocrystalline aluminum thin films. Acta Mater. 2006, 54, 2253-2263. [CrossRef]

37. Zhang, K.; Weertman, J.R.; Eastman, J.A. Rapid stress-driven grain coarsening in nanocrystalline Cu at ambient and cryogenic temperatures. Appl. Phys. Lett. 2005, 87, 061921. [CrossRef]

38. Zhu, Q.; Huang, Q.; Guang, C.; An, X.; Mao, S.X.; Yang, W.; Zhang, Z.; Gao, H.; Zhou, H.; Wang, J. Metallic nanocrystals with low angle grain boundary for controllable plastic reversibility. Nat. Commun. 2020, 11, 3100. [CrossRef]

39. Yuan, Y.; Li, X.; Yang, W. Low-angle grain boundary structures and size effects of nickel nanolaminated structures. J. Mech. Phys. Solids 2019, 130, 280-296. [CrossRef]

40. Mendelev, M.I.; Srolovitz, D.J. Impurity effects on grain boundary migration. Modell. Simul. Mater. Sci. Eng. 2002, 10, R79. [CrossRef]

41. Aramfard, M.; Deng, C. Influences of triple junctions on stress-assisted grain boundary motion in nanocrystalline materials. Modell. Simul. Mater. Sci. Eng. 2014, 22, 055012. [CrossRef]

42. Niu, L.L.; Peng, Q.; Gao, F.; Chen, Z.; Zhang, Y.; Lu, G.H. Effects of interstitial defects on stress-driven grain boundary migration in bcc tungsten. J. Nucl. Mater. 2018, 512, 246-251. [CrossRef]

43. Chen, D.; Xu, S.; Kulkarni, Y. Atomistic mechanism for vacancy-enhanced grain boundary migration. Phys. Rev. Mater. 2020, 4, 33602. [CrossRef] 